\title{
Polio vaccination: past, present and future
}

\author{
Ananda S Bandyopadhyay*,1, Julie Garon², Katherine Seib² \& Walter A Orenstein²
}

\begin{abstract}
Live attenuated oral polio vaccine (OPV) and inactivated polio vaccine (IPV) are the tools being used to achieve eradication of wild polio virus. Because OPV can rarely cause paralysis and generate revertant polio strains, IPV will have to replace OPV after eradication of wild polio virus is certified to sustain eradication of all polioviruses. However, uncertainties remain related to IPV's ability to induce intestinal immunity in populations where fecal-oral transmission is predominant. Although substantial effectiveness and safety data exist on the use and delivery of OPV and IPV, several new research initiatives are currently underway to fill specific knowledge gaps to inform future vaccination policies that would assure polio is eradicated and eradication is maintained.
\end{abstract}

\section{Viruses, vaccines \& disease}

Global eradication of polio is within grasp. Only Pakistan, Nigeria and Afghanistan are currently considered endemic for polio because they have never eliminated indigenous polio viruses. Although the overall reduction in global incidence of cases has been more than $99 \%$ since the eradication efforts begun in 1988 when an estimated 350,000 persons were paralyzed by wild polio viruses (WPVs), there are still a few hundred cases of WPV-related paralysis each year (416 cases in 2013 and 359 in 2014) [1]. These cases are occurring both in the endemic countries as well as in countries re-infected via importations.

Three serotypes exist for polio: poliovirus type 1, poliovirus type 2 and poliovirus type 3 . Wildtype 2 (WPV2) is considered eradicated as the last naturally occurring case was detected in India in 1999. Wild-type 3 (WPV3) appears to be on the verge of eradication with no cases reported since November of 2012, the longest period that a WPV3 has not been isolated.

Two vaccines, live attenuated oral poliovirus vaccine (OPV) and inactivated poliovirus vaccine (IPV) are used throughout the world to protect against polio (Figure 1). In 121 countries (Figure 1B) [2], OPV is used instead of IPV for several reasons: OPV costs substantially less than IPV $(15 \&$ vs US $\$ 1$ or more) [3]; primary immunization with OPV induces superior intestinal immunity compared with IPV and thus has the potential to better prevent transmission of wild viruses; OPV confers contact immunity through passive immunization of unvaccinated persons from viruses shed by vaccines; and OPV is administered in oral drops, which are easier to administer than IPV injections and easier to store and transport.

Despite these advantages, most developed countries have transitioned to IPV, primarily because OPV has the major disadvantage of causing paralytic disease in rare cases [4]. It can

\section{KEYWORDS}

- disease eradication

- inactivated polio vaccine

- polio end game $\bullet$ vaccine technology 
(A)
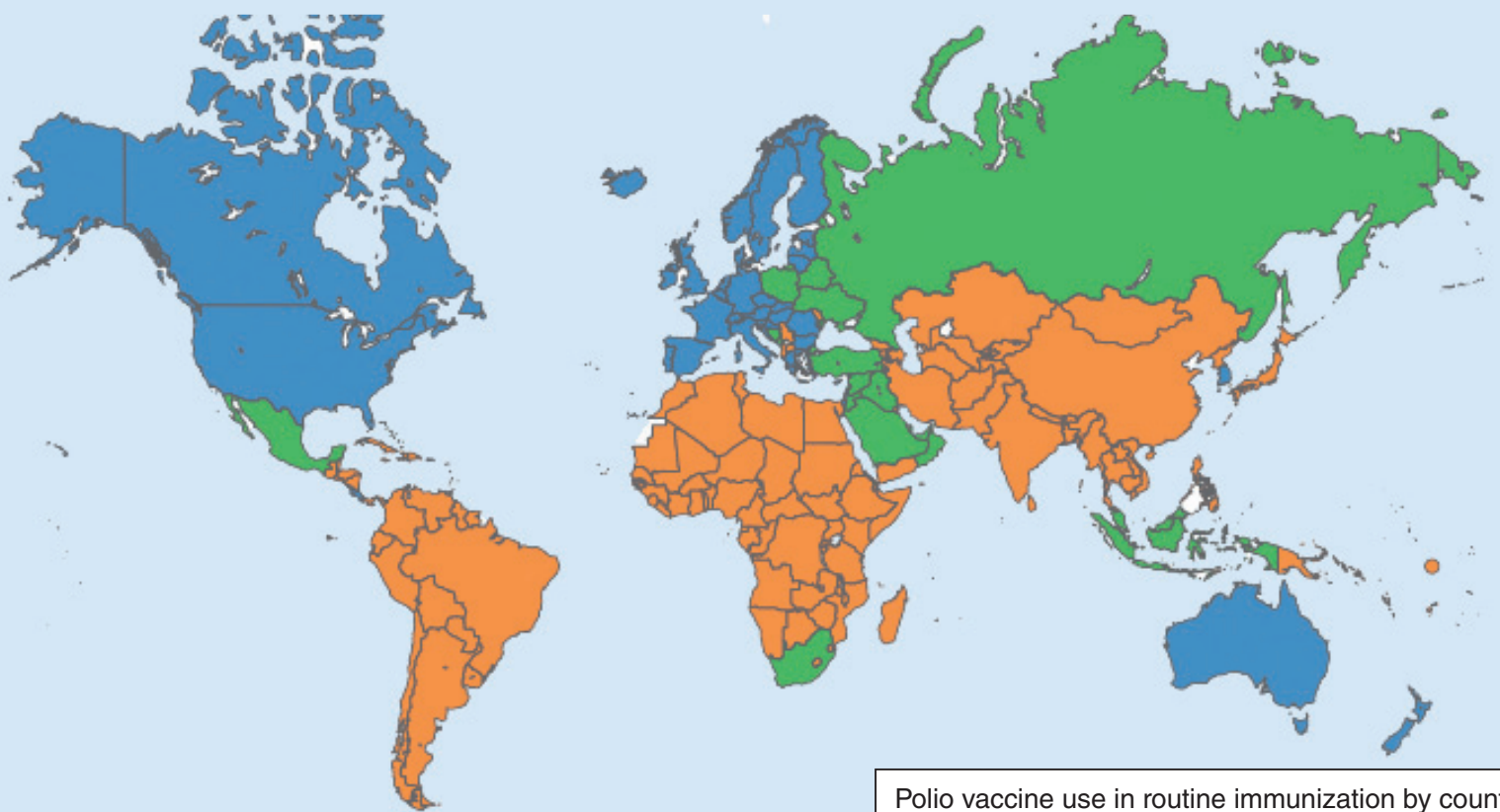

Polio vaccine use in routine immunization by countries $\square$ IPV only, $\mathrm{n}=42$

IPV and OPV (sequential or mixed), $\mathrm{n}=24$

OPV only, $\mathrm{n}=128$

(B)
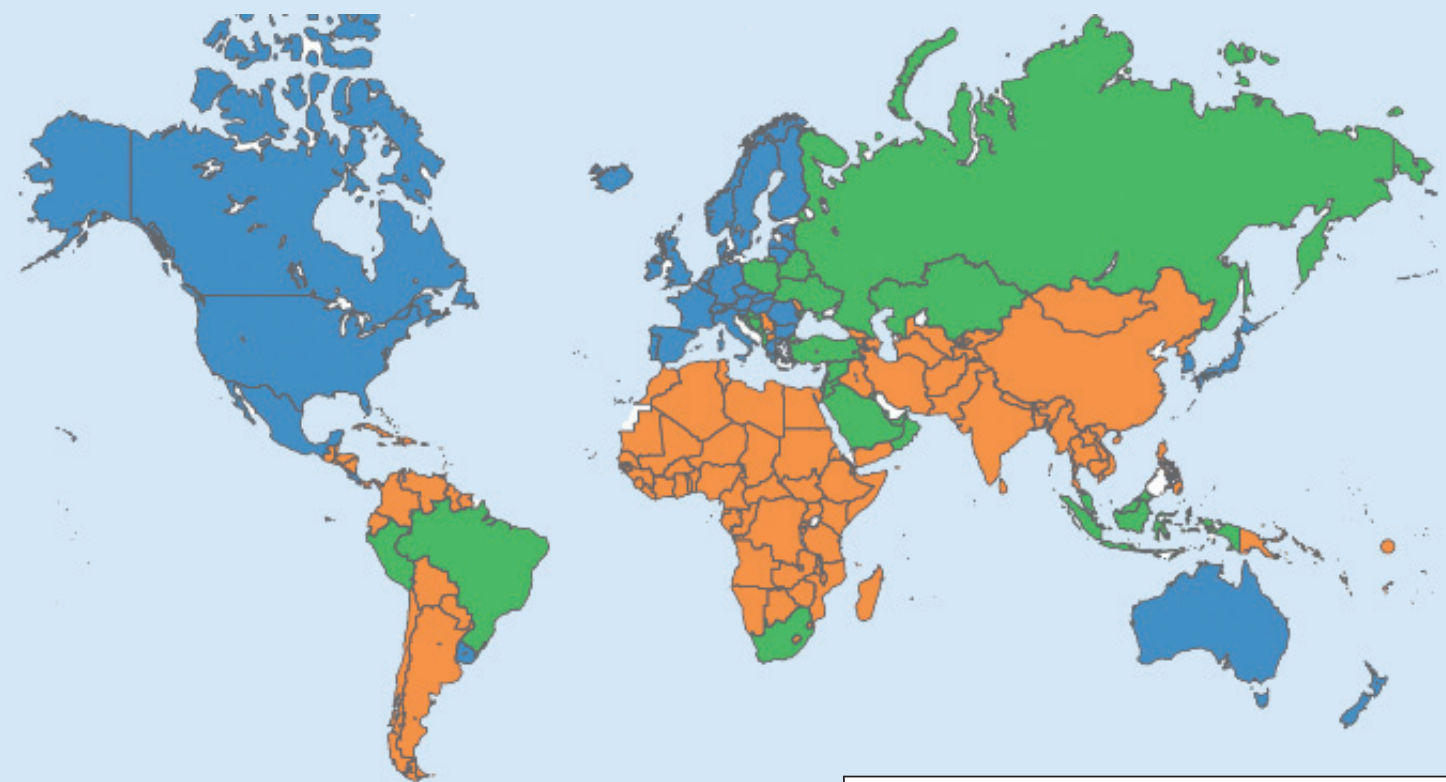

Polio vaccine type data included for WHO countries only. Note: all countries with OPV only or sequential/mixed schedules use tOPV (except Israel, which uses bOPV). 


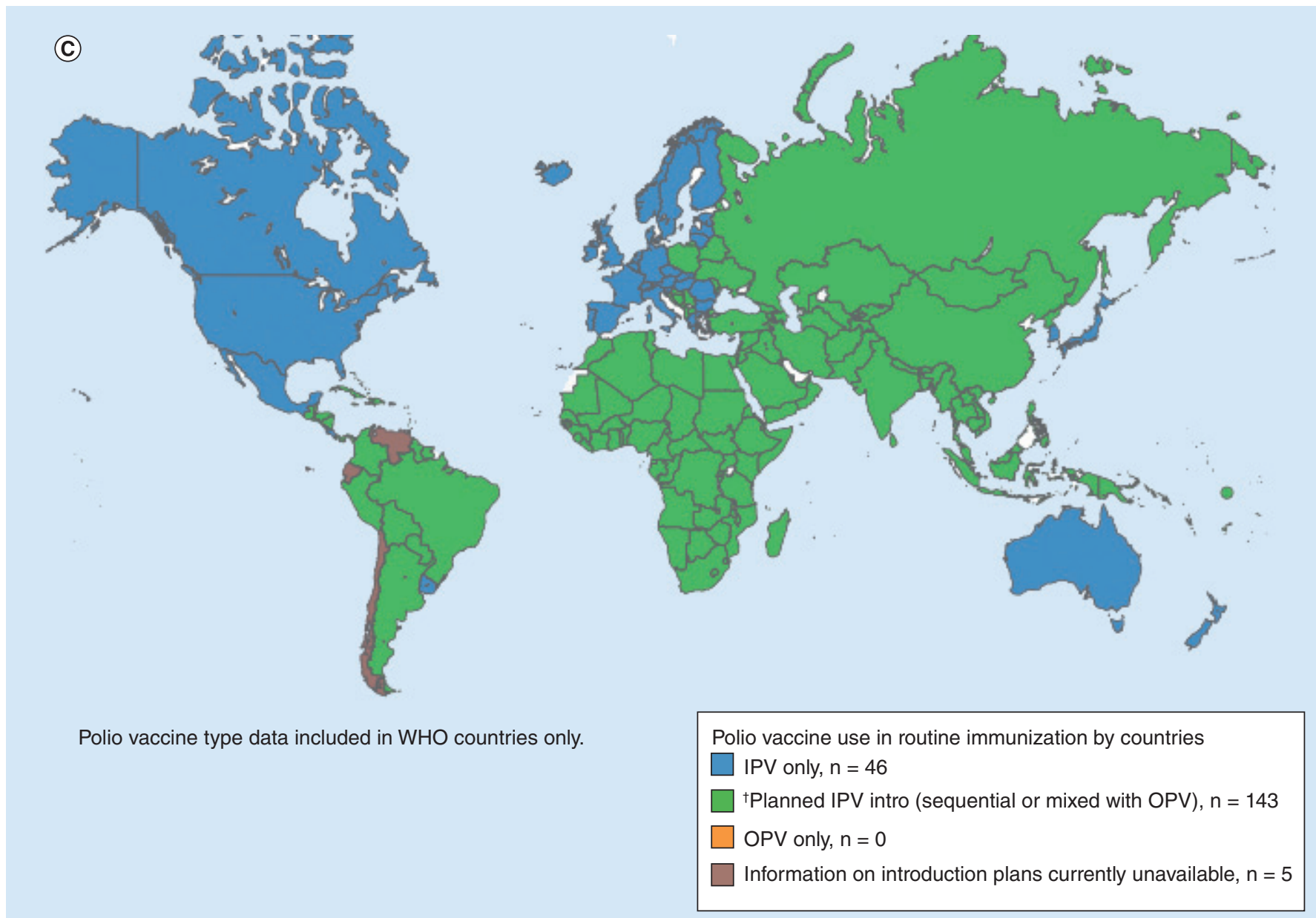

Year: 2016 (projected)

Figure 1. Polio Vaccination: past, present and future (for parts [A \& B], see facing page; for part [C], see above). (A) Polio vaccine use in routine immunization, year 2012. (B) Polio vaccine use in routine immunization, year 2014. (C) Polio vaccine use in routine immunization, year 2016 (projected).

${ }^{\dagger}$ Includes countries that have formally committed to introduce (defined by WHO planning committee as submitting documentation of decision to introduce IPV to and verified by the relevant WHO Regional Office) and those which have indicated intent to introduce. Where specific data on whether new countries introducing IPV will choose IPV only or sequential schedule is pending, these countries are included in the 'Planned IPV Intro' category. All OPV in use in routine immunization from April 2016 is expected to be bOPV. bOPV: Bivalent OPV; IPV: Inactivated polio vaccine; OPV: Oral polio vaccine; tOPV: Trivalent OPV.

(A) Data taken from UNICEF WHO joint reporting form, July 2012. (B) Data taken from UNICEF WHO joint reporting form, July 2014. (C) Data taken from WHO IPV introduction planning status report, November 2014.

cause vaccine-associated paralytic poliomyelitis (VAPP) in vaccine recipients and close contacts at an estimated rate of about 4.7 per million births (range: 2.4-9.7) globally [5]. Typically, the risk of VAPP is highest with the first dose of OPV in industrialized countries - about 6.6-times higher for first-time recipients than those receiving subsequent doses whereas in developing countries the risk of VAPP can be lower for first dose recipients [6]. Data from India show that VAPP is largely associated with second or subsequent doses of OPV, indicating that the age of onset for VAPP is higher in lower-income settings [7]. This is believed to be due, in part, to persisting maternally derived antibodies in the infant when the birth dose is given in areas where wild virus is circulating or circulated recently and because of tropical enteropathies inhibiting vaccine virus from infecting and replicating in the infant. The greatest risk of VAPP is among immunocompromised people with B-cell deficiencies as they carry greater than 3200-times 
the risk of VAPP than the general population [6]. Type 3 virus is the most commonly isolated virus in people with VAPP who do not have immunodeficiencies and type 2 virus is the most commonly isolated virus in those with immunodeficiencies [6]. Risk for VAPP also differs based on receipt of OPV (recipient VAPP) or contact with someone who received OPV (contact VAPP). In 1999 in India, the overall risk of recipient VAPP was 1/12.2 million OPV doses administered while the risk of total VAPP was 1/4.1 million OPV doses administered [7].

Moreover, the live vaccine virus also can mutate in ways that confer the transmissibility and neurovirulence properties of wild viruses, leading to polio outbreaks caused by these altered viruses known as circulating vaccinederived polioviruses (cVDPVs). There have been an average of 76 reported cases of cVDPVs annually between 2005 and 2013 [1]. Regions with increased risk for cVDPVs are those with low vaccination coverage rates, where competing WPVs have been eliminated, and where epidemiologic conditions (e.g., low socioeconomic status, poor hygiene/sanitation and crowding) favor poliovirus transmission [6]. Since 2000, cVDPVs, as defined by association with two or more acute flaccid paralysis cases, have been reported in 23 countries, most of which are developing countries [1].

Due to the historic progress made in interrupting WPV transmission, vaccine viruses are estimated to have caused in 2012 and 2013 more cases of polio-related paralysis (VAPP and VDPVs) globally than were caused by wild viruses creating a unique epidemiological situation with ethical, political and social implications. Therefore, use of OPV will soon have to be phased out in order to eradicate polio from all causes [8].

\section{The polio end game}

Led by WHO, the United Nations Children's Fund (UNICEF), Rotary International and the CDC among other organizations, the Global Polio Eradication Initiative (GPEI) has developed the Polio Eradication and Endgame Strategic Plan that aims to wipe out the last cases of polio from all causes by 2018 [9].

Because type 2 virus accounts for more than $95 \%$ of cVDPV outbreaks detected in recent years and approximately $30 \%$ of VAPP cases [5], a critical intermediate step is to replace the currently used trivalent OPV (tOPV), which protects against types 1, 2 and 3, with a bivalent OPV (bOPV), which protects against types 1 and 3. The plan also calls for the addition of at least one dose of IPV (all currently licensed IPV is trivalent and protects against all types of polio) in routine immunization (RI) programs by 2015 in the OPV-only countries. IPV will provide immunity to a substantial proportion of the population [10] during this proposed OPV switch as well as during complete OPV withdrawal posteradication in case of the possible emergence of type $2 \mathrm{cVDPVs}$, as well as in case of potential breaks in laboratory containment of wild or vaccine viruses. Such a plan is expected to change the landscape of global polio vaccine use in an incredibly short span of time (Figure 1C).

The 2013-2018 strategic plan is expected to cost US $\$ 5.5$ billion over the course of the program with continued investment by countries, but promises to yield up to US $\$ 25$ billion in additional net benefits over the next 20 years. Substantial funding will be required throughout the end game to support core program costs, planned and supplemental immunization activities, surveillance, emergency response and containment. Total eradication of polio will lead to major reductions in public health spending on medical care, vaccine financing and programmatic costs once cases are reduced to zero and control activities can be scaled back [9].

\section{Tools currently available \\ - OPV}

Administration of OPV mimics the immune response to natural exposure to WPV generating both humoral and mucosal immunity [6]. IgM antibody becomes detectable as early as 2-3 days after infection, usually disappearing after 2-3 months, while IgG becomes the predominate antibody and may last for life [6]. The type 2 strain of OPV is immunodominant and in formulating tOPV, the amount of type 2 virus is reduced compared with the other viruses. Most formulations of tOPV have a 10:1:6 ratio of virus for the three serotypes, respectively. In the USA, after a complete primary vaccination series of three doses, over $95 \%$ of recipients seroconvert to all three poliovirus serotypes with long-lasting immunity [6]. However, in developing countries, tOPV has been found to be less immunogenic with an average of 73, 90 and $70 \%$ of children seroconverting to types 1,2 and 3, respectively after three tOPV doses [11]. A comprehensive review of correlates of protection 
against poliovirus found that existence of any titer of homologous neutralizing antibody is protective against clinical paralytic polio in the individual [12].

In a meta-analysis of 31 studies, vaccination with OPV protected individuals against shedding of poliovirus in stool after challenge (failure to shed virus upon vaccine virus challenge is used as a measure of intestinal mucosal immunity) when compared with unvaccinated individuals (summary odds ratio 0.13 with confidence intervals $0.08-0.24)$ [13]. High levels of individual intestinal immunity preventing virus shedding (and therefore transmission) resulting in population protection, along with OPV's ease of administration and low cost contributed to the choice of OPV for WPV elimination in the resource-constrained, high disease burden settings.

There are three formulations of OPVs in use in the world: tOPV containing Sabin strains of all three poliovirus serotypes; bOPV containing Sabin strains for types 1 and 3; and monovalent OPV (mOPV), which has two subtypes for serotypes 1 (mOPV1) and 3 (mOPV3). In addition, monovalent OPV for serotype 2 (mOPV2) is available for research purposes as well as for response procedures should there be an outbreak of poliovirus type 2 . In a randomized, doubleblind, controlled trial in India, the cumulative seroconversion rates to serotype 1 following two doses at birth and at 30 days, were $90 \%$ for $\mathrm{mOPV} 1$ and $86 \%$ for bOPV compared with $63 \%$ for tOPV [14]. For serotype 2, the seroconversion rates were $90 \%$ for mOPV2 compared with $91 \%$ for tOPV, and for poliovirus type 3 , seroconversion rates were $84 \%$ for mOPV3 and $74 \%$ for bOPV compared with $52 \%$ for tOPV [14]. Currently, tOPV is the most commonly used OPV in RI globally (Figure 1B), while bOPV and less commonly tOPV are used in supplemental immunization activities (SIAs). SIAs are mass vaccination campaigns usually targeting children less than 5 years of age regardless of prior vaccination status within a short time frame.

\section{- IPV}

The current formulation of IPV induces close to $100 \%$ seroconversion rates with high antibody titers to the three poliovirus serotypes after a series of three doses, when administered in schedules in which the last dose is administered at 6 months of age or older [15]. However, in a randomized clinical trial in Puerto Rico, study arms vaccinated in a schedule of 6,10 and
14 weeks when compared with the schedule in the USA of 2, 4 and 6 months, had slightly lower seroconversion rates for poliovirus type 1,2 and 3 (85.8, 86.2 and $96.9 \%$, respectively, compared with 99.6, 100 and $99.1 \%$ for the US schedule) [16]. Lower rates of seroconversion are seen in the presence of high levels of maternally derived antibody. Administration of IPV at older ages, giving a chance for maternally derived antibody to wane, is associated with higher levels of seroconversion [16]. Currently accepted correlates of protection for IPV containing vaccines require neutralizing antibody levels at or above a 1:4 to 1:8 dilution threshold [17].

IPV shows a similar effect to OPV in inducing pharyngeal immunity, but has limited effect in inducing primary intestinal immunity when administered alone and thus, IPV is equivalent to OPV in reducing oral shedding following an OPV challenge but is inferior to OPV in reducing intestinal shedding when used for primary immunization in subjects who have not previously been exposed to OPV [18]. In fact, the proportion of persons who shed virus in stool following an OPV challenge in IPV vaccinees is similar to the proportion who shed following a dose of OPV to unvaccinated persons. However, despite shortcomings in induction of intestinal immunity following a primary series, IPV has been shown to reduce both the duration of shedding and the amount of virus shedding in the stool (with a range between studies of 63 and $91 \%$ reduction in the total amount of virus shed). This implies that IPV may reduce transmission even in places where fecal-oral spread is thought to be the predominant mode of transmission [13,19].

IPV alone terminated polio transmission in several Nordic countries of Europe (e.g., Sweden and The Netherlands). It is unclear whether the reason for this success is because oral-oral transmission is the predominant mode of transmission in these countries or if there was additional impact from reduction in quantity and duration of fecal shedding induced by IPV. Whether IPV alone in a developing country population in whom fecal-oral transmission is thought to predominate, can terminate transmission of wild or vaccine viruses is not clear.

After many years (2005-2013) of consistent high coverage using IPV exclusively, Israel recently reported detection of WPV1 from environmental sewage samples throughout the country for over a year (February 2013-March 2014) 
while no symptomatic polio cases were found $[20,21]$. This event has alerted the public health community to the potential for importation and silent transmission in countries using only IPV. Nevertheless, IPV should decrease the incidence of paralytic polio and reduce the spread of polioviruses if they are introduced, compared with an unvaccinated population, by reducing oral-oral transmission and reduced duration of shedding and titer shed.

Since its development in the 1950s, IPV has been one of the safest vaccines in humans, whether used alone or in combination vaccines $[22,23]$. IPV is offered as a standalone vaccine as well as in combination vaccines for primary immunization (ten products) and for boosters (>five products) [18]. Since early in the development of IPV, it has been co-administered with diphtheria, tetanus and pertussis vaccine (DTwP and DTaP), Haemophilus influenzae type $b$ vaccine (Hib) and hepatitis $B$ vaccine.

No serious adverse events have been causally associated with IPV. When used alone, IPV is well tolerated. In one study $5.5 \%$ of injections were accompanied by induration or by erythema [24]. When IPV is administered along with DTP or as a combined DTP-IPV reactogenicity was similar to administration of DTP alone. Rates of local reactions were similar in recipients of either DTP or DTPIPV [24]. A recent review of the US Vaccine Adverse Events Reporting System (VAERS) from 1999 to 2012 also reported that there were no concerning safety issues of adverse events for IPV [25]. A 2014 systematic review of the literature on the safety of routine vaccines recommended for children in the USAfound insufficient evidence to report any association between IPV and sensitivity to food allergens (as one post-licensure study reported) and that serious adverse events are extremely rare for all routine vaccines [26]. Moreover, IPV administered before OPV reduces VAPP cases compared with OPV alone. In 2014, the Global Advisory Committee on Vaccine Safety declared IPV and IPV-containing vaccines as having an excellent safety profile, based on available data [25].

\section{Priming effects \& sequential schedules - IPV before IPV}

Although IPV alone has shown limited effect in inducing primary intestinal mucosal immunity, it has shown promise in the area of priming for a systemic immune response. A single IPV dose generally seroconverts a proportion of vaccinees but induces immune memory (primes) in the majority of the remaining seronegative children. Data on the added protection against paralysis conferred by priming, are not conclusive. In a study from Cuba, among those who did not seroconvert after one dose of IPV, 98\% had a priming response (they developed significant antibody responses within 7 days of subsequent exposure) after one dose of IPV [10]. On the other hand, in a case-control study of a WPV1 outbreak in Senegal, effectiveness against paralytic polio was estimated to be $36 \%$ after one dose and $89 \%$ after two doses, values more compatible with per dose IPV seroconversion rates than with priming rates [27].

\section{- OPV before IPV}

Immunogenicity of IPV when given in an OPVexposed population has been studied extensively. A single dose of IPV administered to infants in Côte D'Ivoire previously vaccinated with a three-dose primary schedule of $\mathrm{tOPV}$ was significantly more effective than an additional dose of tOPV in achieving seroconversion at both 6 and 9 months in subjects who were seronegative after the primary series [28]. More recently, a multiarm trial in Moradabad, India compared a supplemental dose of IPV at 6-9 months to children who had completed a primary series of tOPV plus multiple doses of mOPV, to boosting with a standard and higher potency type 1 mOPV [29]. As in the Côte D'Ivoire study, higher rates of seroconversion in baseline seronegative subjects and increased titers in baseline seropositive subjects were noted in the subjects receiving IPV compared with children who received an additional dose of OPV.

Two recent studies done in India have shown that one dose of IPV given to prior recipients of OPV boosts intestinal immunity for types 1 and 3 [30,31]. In children with history of multiple doses of OPV, the proportion of children excreting the challenge poliovirus was significantly lower for those given a booster dose of IPV followed by a bOPV challenge than those who did not receive any booster prior to the bOPV challenge [31]. The duration of this boost in intestinal immunity is, however, unknown.

A dose of OPV given at birth increases seroconversion rates [32] and provides an opportunity to induce mucosal protection in infants before they are exposed to enteric pathogens. WHO recommends a birth dose of OPV for 
polio-endemic countries and countries at high risk for importation and subsequent spread [33].

\section{- IPV before OPV}

Vaccination regimens employing sequential combinations of IPV and OPV have been utilized in a number of countries, including in the USA. In developed countries where elimination of polio was achieved, VAPP was seen as a major public health problem and resources were available to address the increased costs of using IPV in a sequential IPV to OPV schedule. The intent of this schedule was to acquire the advantages of both IPV and OPV while minimizing adverse reactions: initial immunization with IPV to promote humoral immunity, which gives children protection from VAPP, and subsequent OPV vaccination to induce higher levels of intestinal immunity and maintain population-level protection. In Hungary, a country with a significant problem with VAPP cases [34] a sequential schedule comprising one dose of IPV followed by OPV led to a complete disappearance of VAPP $[35,36]$. Another study in Hungary found that one or three doses of IPV followed by three doses of OPV resulted in individual protection against paralytic poliomyelitis as well as reduced cases of VAPP in the population [36,37]. Additional experiences in India and the USA have shown that receiving IPV before OPV appears to protect against VAPP $[7,34,38]$.

In addition, the sequential schedule of IPV followed by OPV in developed country settings achieved high seroconversion rates, with optimal effect obtained using two doses of IPV followed by two doses of OPV, a regimen that also produced intestinal immunity comparable to three doses of OPV [39]. The efficacy of this strategy has also been studied in the developing world: a trial of IPV followed by OPV in Guatemalan infants demonstrated robust humoral immunity even after only two doses of IPV [40]. Also, use of $\mathrm{mOPV}$ in an outbreak control setting in a population who received a dose of IPV is likely to lead to higher immunity levels than a single dose of mOPV in a completely susceptible population, as the IPV-vaccinated population would already be partially protected with the existence of neutralizing antibodies from previous administration of IPV [39,41].

\section{Vaccine failure: OPV \& IPV}

Several factors can lead to vaccine failure for both OPV and IPV. Environmental and other factors such as malnutrition, concurrent infections, tropical enteropathy (histological changes to the intestinal mucosa) and oral tolerance (suppression of immune responses to prior orally administered antigens) common in developing countries can contribute to reduced mucosal and humoral immunity after OPV vaccination [42]. Also, type 2 vaccine virus in tOPV interferes with responses to OPV types 1 and 3. Additionally, enteric microorganisms and other enteroviruses concurrently present in the intestine at the time of vaccination with tOPV can also interfere with seroconversion to all three serotypes, demonstrating the need for multiple doses, especially in developing countries with poor hygiene [11]. Furthermore, physiological changes and simple kinetics associated with diarrheal states may inhibit poliovirus colonization altogether [11]. A study in India found that immunization with OPV (particularly mOPV) has a significant effect on mucosal immunity although additional doses of OPV have a limited ability to further boost mucosal immunity in children with multiple reported doses of mOPV [43].

For IPV, maternally derived antibody during early infancy has been shown to be the biggest risk factor for lack of vaccine take, and thus vaccine failure [16,44]. Therefore, it is recommended that if only a single dose of IPV is incorporated into the immunization schedule, the dose should be given at 14 weeks of age with DTP3, or first contact after 14 weeks, to ensure good immunogenicity [32]. Recent estimates suggest that the coverage with one dose of IPV at 14 weeks of age or later will be lower compared with administration at earlier ages due to children dropping out of the immunization schedule with increasing age [45]. However, the increase in immunogenicity is thought to outweigh the risk of lower coverage due to high RI dropout rates at higher age groups $[15,46]$.

\section{Enabling eradication: SAGE recommendations}

A polio-free world means complete interruption of transmission of WPVs as well as elimination of all polio disease including VAPP and VDPVs [9]. To achieve this, planning for synchronized cessation of OPV is underway, starting with Sabin type 2. In addition, the Strategic Advisory Group of Experts on Immunization (SAGE) has recommended the introduction of at least one dose of IPV by the end of 2015 to mitigate the risks associated with OPV cessation [32]. 
For countries with a routine Expanded Program on Immunization (EPI) schedule of 6, 10 and 14 weeks and deciding to give only one dose of IPV, that dose should be given at 14 weeks of age, at the same time DTP3 is normally administered. Addition of IPV does not impact the use of OPV at this time, which should be continued. Thus, for a child the dose of IPV would usually be administered with the third dose of OPV for countries without a birth dose of OPV or fourth dose of OPV in countries with a birth dose. OPV-only countries have the flexibility to consider alternative schedules (such as 6, 10 and 14 weeks, 2, 3 and 4 months or 2, 4 and 6 months) and more than one IPV dose but are encouraged to develop a plan for IPV introduction by the end of 2014, based on tiered priority of countries related to risk of cVDPV emergence and spread [32]. Introduction of IPV in RI preceding the global withdrawal of Sabin type 2 is expected to have several important implications:

\section{- Type 2 immunity base}

Resurgence of wild or vaccine-derived type 2 polivovirus after the withdrawal of routine use of Sabin type 2 in vaccines could occur for various reasons such as laboratory containment failure with resultant emergence of type 2 poliovirus, reseeding of the population from the very rare immune-deficient long-term shedders who are incapable of clearing the intestinal infection by vaccine virus by eliciting an immune response and predominantly shed type 2 polio virus, events of bioterrorism or intentional synthesis and release of virus and generation of type 2 cVDPV from previous use of type 2 containing OPV. Introducing IPV before the tOPV to bOPV switch will ensure that a substantial proportion of the population has immunity against type 2 polio after OPV2 cessation and will mitigate the risks associated with it. Studies have shown that seroconversion against type 2 polio after one dose of IPV ranges from 35 to $63 \%$ [15] and in addition, it has been demonstrated that more than $86.9 \%$ of infants who did not seroconvert after a first dose of IPV had a priming immune response as discussed before [10].

\section{- Facilitate interruption of transmission}

Data on the role of IPV in inducing intestinal immunity, as well as IPV's role in reducing transmission in a population setting, remain less clear [47]. Recent poliovirus transmission modeling has suggested that despite better individual seroconversion rates, a complete switch from OPV to IPV could potentially decrease population immunity, especially in settings of intense fecal-oral transmission [48]. As the GPEI progresses toward the eradication of WPVs, national and global health leaders must still actively consider options for managing poliovirus risks, including risks associated with using OPV such as VAPP and VDPVs. The promising data on the impact of IPV in inducing intestinal immunity in an OPV-exposed population and the potential of IPV priming for a robust and accelerated OPV response in case of an outbreak after the OPV2 withdrawal will be important considerations that have to be balanced carefully with operational and financial feasibility $[32,33]$.

\section{- Boost immunity to WPV1 \& WPV3}

Use of IPV in conjunction with continued use of at least two bOPV doses prior to the dose of IPV should boost immunity to types 1 and 3 which should hasten eradication of WPV1 and WPV3 and reduce polio disease caused by type 1 and 3 cVDPVs [32]. This would ensure protection against reintroduction of WPV from other populations. Modeling in post-OPV2 settings in environments with poor sanitation and high transmission have found that IPV coverage must be at least $80 \%$ in order to reduce infectiousness by approximately $80 \%$ [49]. However, an expert review of 66 OPV challenge studies considers IPV an important option in protection against paralysis because it offers relatively higher seroconversion rates in poor hygiene settings compared with OPV [48].

Once IPV introduction is complete and several other global criteria are met, a globally coordinated switch from tOPV to bOPV will take place. The switch will be universal and synchronized to further reduce cVDPV2 and VAPP cases while bolstering eradication of types 1 and 3 . In addition to IPV introduction in RI and prior to the switch, some countries will further boost population immunity to type 2 through additional SIAs with tOPV. Dependent upon progress toward the elimination of persistent cVPDVs, the earliest the switch could take place would be early 2016, after which all tOPV will be removed and destroyed and production of tOPV ceased.

\section{End game barriers}

While it has been shown that IPV integrated into RI programs will indeed reduce the prevalence of paralytic polio within a population, 
uncertainties remain on IPV's role in impacting transmission as part of a global polio eradication strategy as evident with the situation in Israel in 2013-2014 with more than a year of WPV1 isolation in sewage samples as discussed before [21]. On the other hand, Yogyakarta in Indonesia also switched to an all IPV schedule and has not detected any VDPVs since the change [50]. Yogyakarta had very high coverage and improved economic and public health infrastructure which may limit generalizability of this case to the low-income settings. Also, the force of infection of a vaccine virus may be different than that of a WPV, and IPV may be more effective against the former than the latter. It will be important to continue to monitor for circulation of type 2 viruses in particular as IPV becomes the only inducer of immunity to type 2 with the proposed switch of tOPV to bOPV.

\section{Tools of the future}

OPV and IPV are both valuable tools in the polio eradication end game but are not without limitations. As the number of WPV cases continues to decrease and end game procedures are finalized, it is important to consider specific, desired attributes which would further contribute to successes made by OPV and IPV. These considerations will further guide research efforts. The kind of vaccine desired for the end game and posteradication period would ideally have high humoral and intestinal immunity, long duration of protection, low cost, simple administration, widespread safe production, limited waste and heat and freeze stability, among other characteristics. Table 1 describes the benefits and limitations of current vaccines (OPV and IPV) compared with the 'ideal' vaccine candidate. Advances in research in one or more of these areas would greatly contribute to the polio eradication end game and posteradication phase.

\section{- Immunogenicity studies \& sequential regimens with IPV}

In the global polio eradication end game, the cost of IPV will need to be balanced with effectiveness. While there are substantial data evaluating the humoral immunogenicity of one or two doses of IPV, there is less information on the impact of IPV on duration of fecal shedding postchallenge with OPV as well as the average titer shed after one or two IPV doses in a naive population. This is particularly important with regard to type 2 virus since the one or two doses of IPV would be the sole source of immunity to that serotype a child would receive after the switch from tOPV to bOPV. The majority of earlier studies examining fecal excretion have not used explicitly quantifiable methods. Development of correlates of intestinal immunity, if possible, would facilitate testing of new IPV candidates because they would allow assessment of intestinal immunity without the need for challenge studies. In the absence of correlates, challenge studies after the switch would have to be conducted under extreme oversight to prevent sustained transmission if they

Table 1. 'Ideal vaccine' characteristics compared with current tools.

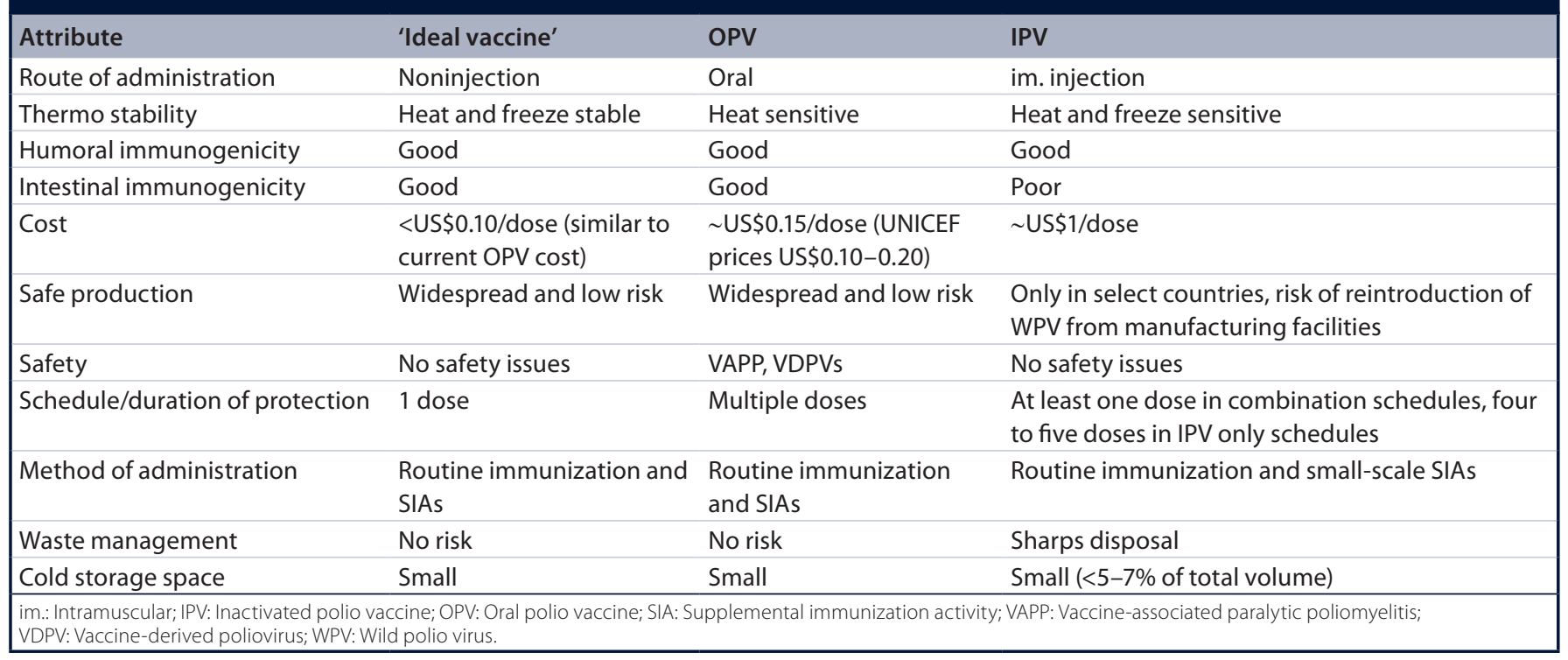


can be conducted at all. The most quantifiable measure of fecal poliovirus excretion is an index that measures viral excretion over a period of time after an OPV challenge [6]. Also, most of earlier studies examining priming and immunogenicity in different schedules were done with schedules involving tOPV, and data related to schedules with bOPV (preceded or followed by IPV) are lacking. Potential benefits of supplemental IPV in the context of the end game include achieving protection against type $2 \mathrm{cVDPV}$ following the transition from tOPV to bOPV, eventual protection from $\mathrm{cVDPV}$ s for all three serotypes during the process of OPV cessation, and prevention of VAPP - particularly after wild-type paralytic polio is eliminated. However, research regarding the optimal strategy (especially relating to timing and number of IPV doses) for utilization of IPV with bOPV in the polio eradication end game is incomplete. Currently, a series of polio vaccine clinical trials (Table 2) funded by the GPEI partners such as WHO, CDC and the Bill and Melinda Gates Foundation are being implemented to assess the impact of full or fractional doses of IPV on primary immunogenicity, priming and viral shedding through various administration regimens. For example, the ongoing clinical trials in South and Central American countries are designed to explore immunogenicity of sequential and concomitant schedules with IPV and bOPV with a type 2 oral vaccine challenge that will allow the assessment of the shedding index for type 2 virus, taking into account the proportion shedding, titer shed and the duration of shedding [51,52]. These studies, along with additional studies being implemented in other parts of the world [53,54] with IPV and bOPV sequential or mixed regimens, are expected to yield results by 2015, and should provide important information on a range of issues related to IPV use including but not limited to timing [55], sequence [51], dosage and delivery route [54,56] that would guide global vaccination policies during the tOPV to bOPV transition in 2016 and beyond. An important consideration to note, however, is the fact that most of these studies are being implemented in a background of tOPV use in RI in the countries, and thus passive exposure of study subjects to Sabin type 2 should be taken into account in the interpretation of study findings.

\section{- Monovalent IPV-2}

Currently available IPV is a formalin inactivated trivalent vaccine comprising the three poliovirus serotypes, type 1, 2, 3 and is manufactured based on enhancements of methods developed in the 1950s by Jonas Salk. Following the development of the $\mathrm{D}$-antigen $(\mathrm{D}-\mathrm{Ag})$ potency assays in the 1970s, the D-Ag content of 40, 8 and $32 \mathrm{D}-\mathrm{Ag}$ units for poliovirus type 1,2 and 3, respectively for current IPV was established based on the historic immunogenicity studies by Salk [57-59] . Based on the data from the dose ranging studies and as a compromise between protective immune response in children and the quantity of vaccine that could be optimally manufactured with cost considerations, WHO defined in 1981 the 40-8-32 D-Ag units composition as the specification for the antigenic content for all trivalent IPV formulations [60].

Under the best of circumstances if only a single dose of IPV is used at 4 months of age, a study in Cuba found only $63 \%$ of recipients seroconverted to type 2 . A higher antigen content monovalent IPV type 2 (m-IPV2) could be an option for the polio end game immunization strategies as a source of more effective primary immunogenicity against type 2 , particularly during the period when bOPV would be used in RI. For this purpose, Bilthoven Biologicals has formulated the m-IPV2 with an antigen content (type 2) of $32 \mathrm{D}-\mathrm{Ag}$ units, four-times the current dose of type 2 in the trivalent IPV. A Phase I study on safety in adults with $\mathrm{m}$-IPV2 is now completed, and a Phase II study on safety and immunogenicity in infants is underway with this product (Table 3). Results from these studies are expected to be available by 2015 , and should provide important information on any potential role of m-IPV2 in the polio eradication end game $[61,62]$.

\section{- Adjuvanted IPV}

To support incorporation of IPV into global immunization programs, a range of approaches are being supported with goals of reducing the cost and increasing the supply of IPV. This includes developing formulations of IPV combined with an adjuvant to improve the immune response and decrease the amount of polio antigens needed. Adjuvants have been shown to influence factors such as onset, magnitude, duration and/or quality of the immune response [77,78]. Such enhanced immune responses could translate into reduction in antigen dose or in the number of immunizations required, which could translate into the reduction of the costs of vaccination. Some adjuvants have also been shown to induce different types of immune responses, such as the 
Table 2. Ongoing research (selected) on current tools.

\begin{tabular}{|c|c|c|}
\hline Location of study & Questions related to current tools & Ref. \\
\hline $\begin{array}{l}\text { Colombia-Dominican Republic- } \\
\text { Guatemala-Panama, India, Bangladesh }\end{array}$ & $\begin{array}{l}\text { Immunogenicity (humoral and intestinal) of } \geq 1 \text { IPV dose combined with bOPV in } \\
6-10-14-\text { week mixed schedules }\end{array}$ & {$[52-54]$} \\
\hline Chile & $\begin{array}{l}\text { Immunogenicity (humoral and intestinal) of IPV } \rightarrow \text { bOPV in 2-4-6-month sequential } \\
\text { regimen }\end{array}$ & [51] \\
\hline Gambia, Bangladesh & Immunogenicity with fractional IPV doses via ID route & {$[54,56]$} \\
\hline India & $\begin{array}{l}\text { Duration of protection of boosting of intestinal immunity induced by IPV in OPV } \\
\text { primed populations }\end{array}$ & [55] \\
\hline
\end{tabular}

enhancement of intestinal immune responses [79], which would improve the chances of interrupting transmission of both WPVs and VDPVs.

In light of the increasing demand for IPV to be used to boost humoral immunity in individuals to protect against paralytic disease during and after the planned switch from tOPV to bOPV by 2016 and all OPV cessation by 2019, IPV adjuvantation strategies have been prioritized. A high priority has been given to efforts to evaluate and, if warranted, to clinically advance IPV formulations adjuvanted with aluminum salts, given that aluminum adjuvants have been shown to promote dose-sparing, have a broad safety database and are already widely used in childhood vaccines, including IPV containing combination vaccines (Table 3). Furthermore, regulators have already approved aluminum as an adjuvant for other vaccines such as DTP, having considered aluminum salt adjuvants as both safe and effective. Planning for clinical studies to evaluate such a formulation with Salk IPV is currently ongoing with funding support from the Bill and Melinda Gates Foundation. As another example, an aluminum hydroxide-adjuvanted Sabin IPV has also recently been clinically evaluated [63].

The development of IPV adjuvanted with novel adjuvants that may enhance intestinal mucosal immunogenicity is also being explored as an important risk-mitigating strategy because some of the adjuvants, such as double-mutant heatlabile enterotoxin ('dmLT'), have been shown in studies conducted with other vaccine formulations to enhance mucosal immune responses in the intestine $[80,81]$. The hope is that the adjuvanted IPV would have the potential not only to prevent paralytic disease (which can already be accomplished with the current unadjuvanted IPV formulations) but also to significantly enhance intestinal mucosal immunity and thereby reduce the risk for shedding and environmental transmission of polioviruses. Due to the anticipated more complex and thus, longer regulatory pathway required for a formulation involving a novel adjuvant such as dMLT, the regulatory approval timeline of such an IPV product would be anticipated to be several years after OPV cessation.

\section{- Novel routes of administration}

Better understanding of the feasibility of IPV use with different modes of administration and concomitantly with other childhood vaccines that have the potential to be used in SIAs are crucial research issues. The intradermal (ID) route of vaccine administration stimulates the immune response by delivery of vaccine antigen directly to dermal dendritic cells, the antigenpresenting cells that drive the generation of follicular T-helper cells and the subsequent activation and isotype-switching of naive B cells [82]. The ID route of administration allows a fractional dose ( $1 / 5$ th of the antigen used in intramuscular [im.] administration) to elicit a similar immune response and therefore has the potential to reduce the cost of vaccination with IPV. Studies conducted to date with one, two and three IPV doses (and a booster dose in the Philippines study) have confirmed the immunogenicity of fractional dose ID delivery [29,83-88]. However, although some studies have reported that seroconversion rates following ID administration of IPV for primary series or priming are lower but noninferior to rates after a standard dose of im. IPV, other studies have found that seroconversion rates after ID administration of IPV have been inferior. Further, geometric mean titers and in some cases median titers induced by ID administration were consistently lower in all studies [29,83-88].

The benefits of disposable-syringe jet injector (DSJI) approach compared with needle and syringe relate to ease of IPV delivery into the skin. The introduction of such an approach into programs in place of im. or ID administered IPV would require more quantitative information 


\section{Table 3. Research (selected) underway on potential new tools for future use.}

\begin{tabular}{|c|c|c|}
\hline Research question/tool & Importance & Status \\
\hline \multirow[t]{2}{*}{$\begin{array}{l}\text { Immunogenicity of monovalent IPV-2 } \\
\text { (m-IPV2) }\end{array}$} & $\begin{array}{l}\text { Improved humoral and intestinal } \\
\text { immunogenicity }\end{array}$ & Phase I (Belgium) study on safety completed [62] \\
\hline & Reduced cost with one dose of m-IPV2 & $\begin{array}{l}\text { Phase II (Panama) study underway to evaluate safety } \\
\text { and immunogenicity [61] }\end{array}$ \\
\hline \multirow[t]{3}{*}{ Immunogenicity of IPV adjuvants } & Reduced cost & $\begin{array}{l}\text { Clinical studies being planned to evaluate potential } \\
\text { for aluminum salts adjuvants and for } \mathrm{dmLT} \\
\text { adjuvants for IPV [63] }\end{array}$ \\
\hline & Increased supply & \\
\hline & Potential for enhanced mucosal immunity & \\
\hline \multirow[t]{3}{*}{$\begin{array}{l}\text { Feasibility of novel routes of } \\
\text { administration }\end{array}$} & Operational advantages & $\begin{array}{l}\text { Studies in humans being planned or implemented } \\
\text { to evaluate impact of im. and ID delivery of } \\
\text { IPV through use of disposable jet injectors, } \\
\text { micro needle patch and other novel delivery } \\
\text { techniques }[56,64-66]\end{array}$ \\
\hline & Potential for use in SIAs & \\
\hline & Concomitant use with other vaccines & \\
\hline \multirow[t]{2}{*}{$\begin{array}{l}\text { Development of genetically stable } \\
\text { OPV and attenuated IPV seed strains }\end{array}$} & $\begin{array}{l}\text { Potential use in outbreak control (reduced risk } \\
\text { of VDPVs and VAPP) or in routine immunization } \\
\text { if IPV is considered inadequate in reducing } \\
\text { transmission risk }\end{array}$ & In preclinical development or planning phase [67-75] \\
\hline & $\begin{array}{l}\text { Further attenuated and less infectious seed } \\
\text { strains for safe IPV manufacture }\end{array}$ & \\
\hline \multirow[t]{2}{*}{ Immunogenicity with Sabin IPV } & $\begin{array}{l}\text { Minimize risk of reintroduction of WPV from } \\
\text { IPV manufacturing facilities }\end{array}$ & Sabin IPV has been licensed in Japan [76] \\
\hline & & $\begin{array}{l}\text { Efficacy and feasibility of large-scale production are } \\
\text { currently being evaluated }[75,76]\end{array}$ \\
\hline
\end{tabular}

on immunogenicity using the devices, training costs of staff on how to properly administer vaccine, and studies on how well those staff use the device. Studies are ongoing in different parts of the world, including a clinical trial in The Gambia [56] that are evaluating the comparative impact of both im. and ID approaches of delivering IPV with DSJI that could potentially facilitate outreach or campaign use of IPV in hard to access areas. Additional clarity on global policy on indications of IPV use (electively in RI, or in outbreak response or in catch up campaigns), scale of investment based on the potential use and regulatory process with regard to ID or im. DSJIs would inform the value proposition of IPV via DSJI in the future.

ID delivery of IPV via microneedles could achieve ease of administration and potentially improve immunogenicity compared with needle and syringe and/or DSJI devices. Several developers are advancing microneedle technology which has the potential to facilitate house-to-house vaccination campaigns for outbreak response by alleviating the need for injections and for trained health staff, reducing wastage and biohazard risks and increasing the capacity for mass vaccination. A patch under development at Georgia Institute of Technology (GA, USA) contains 100 microneedles of less than a millimeter in length which are attached to a flexible pad that is smaller than a postage stamp [64]. Upon application of the patch, the needles puncture the skin and dissolve in 5-10 min, followed by the release of the antigen [64,89]. Experiments with the Georgia Institute of Technology patch and other dermal patches under development demonstrate immune responses comparable with the standard modes of injectable delivery $[64,90,91]$. There has also been considerable interest in exploring additional vaccine-delivery innovations such as sustainedrelease formulations and skin permeabilization techniques which could potentially be applicable for IPV and substantially reduce logistical challenges of IPV administration [65,66,92]. Proof-ofconcept information in humans with such devices and methods should be the next logical step, and 
such data would be important for future incorporation of these innovative tools into the eradication program (Table 3). As with the application of other approaches, cost and regulatory approvals would also be important factors.

\section{- New OPV formulations}

Vaccination against polio is likely to continue for at least 5 years after cessation of all OPV to ensure that both WPV and VDPV are extinct, and do not re-establish circulation. Current plans are to use mOPV2 in SIAs to control outbreaks should a WPV2 or cVDPV2 be found to be circulating in the posteradication era. A new OPV-2 that is genetically more stable than Sabin type 2 could avert the unwanted potential for generating new type $2 \mathrm{cVDPVs}$ with mass use of mOPV2, by significantly reducing the risk of neurovirulence and transmissiblilty. Several approaches are being undertaken to develop new and safer type 2 OPVs that are significantly less likely to cause VAPP or cVDPV. Some of the concepts being explored alone or in combination include [67-75]:

- Stabilizing the Sabin-2 attenuation phenotype by modifying the nucleotides in the 5'UTR;

- Ensuring the maintenance of the modified 5'UTR or increasing attenuation by relocation of a genetic element (cre), which is required for replication, to the $5^{\prime} \mathrm{UTR}$ of the viral genome;

- Reducing the rate of mutation in the viral genome through the selection of mutations that increase the fidelity of RNA-dependent RNA polymerase;

- Attenuating the virus by modifying the nucleotide sequence of the viral capsid by a method called codon deoptimization such that the amino acid sequence of the viral polyprotein is unchanged.

Successful development of a genetically stable type 2 OPV could pave the way for developing genetically stable vaccines against serotypes 1 and 3. If a new OPV-2 could be successfully developed, it would be an important tool for the control and elimination of cVDPVs in the concluding phases of the polio eradication initiative, and beyond (Table 3 ).

\section{- Sabin IPV}

As the global eradication program moves closer to the achievement of zero virus transmission, and cessation of all OPV use, production facilities for IPV would possibly be the only source of WPVs. Therefore, an additional measure to minimize the risk of reintroduction of WPV from IPV manufacturing facilities would be to develop IPVs that are formulated from an attenuated live poliovirus [76]. IPV is usually made from WPV strains such as Mahoney (Salk type 1), MEF-1 (Salk type 2) and Saukett (Salk type 3) which are grown in Vero cell culture or in human diploid cells [93].

Successful development of IPV based on the attenuated Sabin virus strains has led to the licensure of the Sabin IPV in Japan and subsequent introduction of DTP-Sabin IPV formulations in routine immunization program in the country [93]. Although still a risk, Sabin polioviruses are much less likely to cause problems if released accidentally from a production laboratory given that they are less virulent and less transmissible than WPVs. However, the costs, efficacy and feasibility of large-scale production are currently being evaluated, and further research to explore operational and immunological impact of Sabin IPV and standardization of assessment of antigenic content would be important to inform near- and posteradication vaccination policies (Table 3) $[76,93]$.

\section{Conclusion}

Poliovirus transmission has been wiped out from more than $99 \%$ of the world with the successful use of OPV and IPV over the past several decades and effective programmatic use and delivery of these two vaccines in the current polio reservoirs should be adequate to enable global polio eradication in the near future. However, to maintain a world permanently free from the risk of all polioviruses, the eradication program will have to overcome challenges such as the rare occurrence of revertant neurovirulent and highly transmissible strains of polioviruses from OPV, and the relative lack of primary intestinal mucosal protection from IPV. Ongoing and future research initiatives focused on evaluating immunogenicity and safety of current and new vaccine choices in different schedules with careful consideration towards cost and programmatic feasibility have the potential to further accelerate the goal of achieving and sustaining eradication.

\section{Future perspective}

Although poliovirus transmission is at its lowest levels both in terms of geographic foci of persistent circulation and genetic diversity of the circulating strains, significant effort is needed 
to secure the end game, including best use of existing vaccines and adoption of new tools and strategies. As WPV cases decrease, elimination of cVDPV cases will become a priority necessitating the need for withdrawal of OPV-2 and eventual cessation of all OPV use. For many reasons previously described, IPV will need to be introduced globally, and at an unprecedented pace. Although the body of evidence supporting the recommendations for inclusion of IPV in the global end game strategy is extensive, many questions remain unanswered with regard to IPV's role in interrupting transmission in developing countries. To understand if the current and proposed new IPV formulations would have sufficient impact on the transmission of polioviruses, it is critical to take advantage of the opportunity afforded by countries switching from OPV to IPV by conducting ecologic studies and by enhancing environmental poliovirus surveillance to observe if polioviruses continue to circulate, and if the environmental surveillance system is sensitive enough to detect such circulation. Also, more details regarding optimum doses, timing and schedules are needed as countries make decisions based on specialized situations and epidemiology.

Additionally, major barriers to timely introduction of IPV by low- and middle-income countries include vaccine cost, manufacturing capacity to meet demand, cold chain capacity and logistics. Many countries considering IPV introduction have high burden of other infectious diseases and competing health care priorities. This poses unique sociopolitical challenges including the risk of reduced motivation for aggressive interventions against polio, a diminishingly visible disease. For these reasons, innovative vaccine delivery methods and more effective schedules are needed on an urgent basis to secure the gains achieved thus far. In the next 5-10 years, as technology develops in the field of vaccines overall, huge advances are expected to be made in the areas of safety, efficacy and ease of vaccine administration. A future in immunization, with vaccines having an everexpanding antigen profile, would likely see combination vaccines with IPV, high efficacy vaccines not requiring boosters, more heat-stable vaccines and vaccines requiring very little technical skill to administer (e.g., patches, among others).

In the past, combination vaccines have been developed containing both IPV and DTwP, but it is important to note that problems with the coformulation arose due to the denaturing of IPV by the residual thimerosal used to manufacture many whole cell vaccines [18]. IPV and DTwP can, however, be administered concomitantly via separate injections. Additionally, SAGE recommends continued use of whole cell pertussis vaccines in developing countries due to the lower initial efficacy, faster waning of immunity and potentially reduced impact on disease transmission associated with acellular pertussis vaccine relative to the currently available whole-cell vaccines [94]. Thus, further research is needed to explore the viability of a successful whole cell pertussis based construct of a hexavalent vaccine containing IPV, and if such an approach is successful and affordable, there could be significant gains in immunogenicity against polio and program implementation with a three-dose hexavalent schedule.

The substantial resources being allocated to the final stages of polio eradication reflect the collaborative effort of the global health community in rising to the challenge of bringing technical and operational innovations to the most difficult to reach places of the world. After the last polioviruses have disappeared, the legacy of the epic battle of polio eradication has the potential to guide countless public health efforts underway to ensure a healthier future for the world's children.

\section{Acknowledgements}

The authors would like to thank the following: Bill and Melinda Gates Foundation (staff and consultants): Deborah Lans, Amy Weiner, John F Modlin, Jay Wenger, Penny Heaton, Chris Wilson, Richard Wilder and Tasleem Kachra, Julian Fellerman, Feyrouz Kurji, Khatuna Giorgadze and Robin Schofield; researchers and sponsors of ongoing and recently completed polio vaccine trials: Edwin J Asturias, Ricardo Rüttimann, Miguel O'Ryan Gallardo, Jose Jimeno, Xavier Saez Llorens, Ed Clarke, Beate Kampmann, Geert Leroux-Roels, Ralf Clemens, Sue Ann Clemens, Abhijeet Anand, Roland W Sutter and Jacob John.

\section{Disclaimer}

Although some of the studies reported in this review were supported by grants from $B M G F$, the review findings discussed in the article are independent of this interest.

\section{Financial \& competing interests disclosure}

AS Bandyopadhyay is an employee at the Bill and Melinda Gates Foundation (BMGF). J Garon, K Seib and WA Orenstein receive salary support through a BMGF grant awarded to the Task Force for Global Health. WA Orenstein serves as a consultant to the BMGF. The authors have no other relevant affliations or financial involvement with any organization or entity with a 
financial interest in or financial conflict with the subject matter or materials discussed in the manuscript apart from those disclosed.

No writing assistance was utilized in the production of this manuscript.

\section{Open access}

This work is licensed under the Creative Commons

Attribution-NonCommercial 3.0 Unported License. To

view a copy of this license, visit http://creativecommons.org/

licenses/by-nc-nd/3.0/

\section{EXECUTIVE SUMMARY}

\section{Viruses, vaccines \& disease}

- Wild poliovirus cases (types 1,2 and 3) have decreased by $99 \%$ since the 1988 World Health Assembly resolution to eradicate polio.

- Oral polio vaccine (OPV) is the tool generally used in the eradication effort due to low cost, ease of administration and community protection, but rarely, paralysis due to vaccine can result.

- Inactivated polio vaccine (IPV), used mostly in developed countries for routine immunization (RI), induces high individual protection against paralysis but in countries in which fecal-oral transmission is thought to be dominant provides lower protection against transmission of polioviruses because induction of intestinal immunity by IPV is inferior to OPV.

\section{The polio end game}

- The Strategic Advisory Group of Experts (SAGE) recommends all OPV using countries introduce at least one dose of IPV into the RI schedule before the end of 2015 in order to provide a poliovirus type 2 immunity base prior to and after the global switch from trivalent OPV to bivalent OPV (bOPV).

\section{Tools currently available}

- IPV is a very safe vaccine and when used in sequential or combination schedules with OPV can reduce the prevalence of vaccine-associated paralytic poliomyelitis and protect against emergence of type 2 poliovirus.

- Environmental factors such as malnutrition, concurrent infections and tropical enteropathy can lead to OPV vaccine failure while interference by maternally derived antibodies are the primary cause of vaccine failure for IPV.

\section{Enabling eradication: SAGE recommendations}

- SAGE recommends at least one dose of IPV prior to the trivalent OPV to bOPV switch in order to reduce risks associated with OPV 2 cessation; facilitate interruption of transmission with the use of monovalent OPV2 if type 2 outbreaks occur; and boost immunity against types 1 and 3 thus hastening polio eradication.

\section{End game barriers}

- Uncertainties remain on IPV's role as part of a global polio eradication strategy in impacting on transmission in developing countries with largely fecal-oral transmission.

- The high cost and operational issues of IPV use pose a threat to timely introduction in developing countries that have many competing public health priorities.

\section{Tools of the future}

- Research is underway to assess the impact of the number and timing of full or fractional doses of IPV on priming and viral shedding.

- Several new vaccine technologies are currently being explored such as monovalent IPV-2, aluminum salt adjuvants, double-mutant heat-labile enterotoxin adjuvants, intradermal delivery of IPV with innovative tools, IPV manufactured from Sabin strains and more genetically stable OPV strains among other research initiatives.

\section{Future perspective}

- Significant effort is required to rid the world of the last reservoirs of poliovirus disease and secure eradication for generations to come. Although the currently available vaccines are effective, safe and have been proved to be adequate in eliminating the disease from most part of the world, improved tools and techniques can further accelerate the process of achieving and sustaining eradication in developing countries. 


\section{References}

Papers of special note have been highlighted as:

- of interest; $\bullet$ of considerable interest

1 The Global Polio Eradication Initiative. Data Monit.

www.polioeradication.org

- Contains case counts and surveillance data, updated weekly.

2 World Health Organization. Immunization schedules by antigens. Data Stat. Graph. http://apps.who.int/immunization

3 UNICEF. Current IPV supply, recent tender results \& outlook for the future. (2012). www.unicef.org/supply/files/IPV_Supply

4 Aylward B, Tangermann R. The global polio eradication initiative: lessons learned and prospects for success. Vaccine 29 (Suppl. 4), D80-D85 (2011).

- Discusses progress resulting from development of the Global Polio Eradication Initiative strategic plan as well as challenges encountered, approaches to overcome them and planning toward the polio end game.

5 Platt LR, Estívariz CF, Sutter RW. Vaccineassociated paralytic poliomyelitis: a review of the epidemiology and estimation of the global burden. J. Infect. Dis. 210 (Suppl. 1), S380-S389 (2014).

- Provides updated global estimates on vaccine-associated paralytic polio taking into account developing country epidemiology.

6 Sutter RW, Kew OM, Cochi SL, Aylward RB. Poliovirus vaccine - live, Table 28-12: Intestinal Immunity in Vaccinated (OPV or IPV) and Naturally Immune and Susceptible Children. In: Vaccines (6th Edition). Plotkin SA, Orenstein WA, Offit PA (Eds). W.B. Saunders, London, UK, 623-624 (2013).

- Chapter in 'Vaccines' textbook provides information on poliomyelitis, epidemiology, vaccine development, oral poliovirus vaccine (OPV) characteristics and programmatic implementation of the polio end game.

7 Kohler KA, Banerjee K, Gary Hlady W, Andrus JK, Sutter RW. Vaccine-associated paralytic poliomyelitis in India during 1999: decreased risk despite massive use of oral polio vaccine. Bull. World Health Organ. 80(3), 210-216 (2002).

8 Aylward B, Yamada T. The polio endgame. N. Engl. J. Med. 364(24), 2273-2275 (2011).

9 Global Polio Eradication Initiative. Polio eradication \& endgame strategic plan 2013-2018 (2013).

www.polioeradication.org/Portals
-• Global Polio Eradication Initiative's comprehensive, long-term strategy that addresses eradication of all polio disease including wild poliovirus and circulating vaccine-derived poliovirus.

10 Resik S, Tejeda A, Sutter RW et al. Priming after a fractional dose of inactivated poliovirus vaccine. N. Engl. J. Med. 368(5), 416-424 (2013).

11 Patriarca PA, Wright PF, John TJ. Factors affecting the immunogenicity of oral poliovirus vaccine in developing countries: review. Rev. Infect. Dis. 13(5), 926-939 (1991).

12 Sutter RW, Pallansch MA, Sawyer LA, Cochi SL, Hadler SC. Defining surrogate serologic tests with respect to predicting protective vaccine efficacy: poliovirus vaccination. Ann. NY Acad. Sci.754(1), 289-299 (1995).

13 Hird TR, Grassly NC. Systematic review of mucosal immunity induced by oral and inactivated poliovirus vaccines against virus shedding following oral poliovirus challenge. PLoS Pathog. 8(4), e1002599 (2012).

- Review of 31 studies recording the prevalence of poliovirus shedding in stool or nasopharyngeal samples after a challenge dose of OPV.

14 Sutter RW, John TJ, Jain H et al. Immunogenicity of bivalent types 1 and 3 oral poliovirus vaccine: a randomised, double-blind, controlled trial. Lancet 376(9753), 1682-1688 (2010).

15 Estivariz CF, Pallansch MA, Anand A et al. Poliovirus vaccination options for achieving eradication and securing the endgame. Curr. Opin. Virol. 3(3), 309-315 (2013).

- Describes past, current and future use of OPV and inactivated poliovirus vaccine as well as additional research underway to support the polio end game.

16 Dayan GH, Thorley M, Yamamura Y et al. Serologic response to inactivated poliovirus vaccine: a randomized clinical trial comparing 2 vaccination schedules in Puerto Rico. J. Infect. Dis. 195(1), 12-20 (2007).

17 Plotkin SA. Correlates of protection induced by vaccination. Clin. Vaccine Immunol. 17(7), 1055-1065 (2010).

18 Vidor E. Poliovirus vaccine-inactivated. In: Vaccines (6th Edition). Vidor E, Stanley A (Eds). Plotkin Elsevier/Saunders, PA, USA, 573-597 (2013).

19 Laassri M, Lottenbach K, Belshe R et al. Effect of different vaccination schedules on excretion of oral poliovirus vaccine strains. J. Infect. Dis. 192(12), 2092-2098 (2005).
20 Anis E, Kopel E, Singer S et al. Insidious reintroduction of wild poliovirus into Israel, 2013. Euro Surveill. 18(38), pii: 20586 (2013).

21 Kopel E, Kaliner E, Grotto I. Lessons from a public health emergency - importation of wild Poliovirus to Israel. N. Engl. J. Med. 371(11), 981-983 (2014).

22 Poliomyelitis prevention in the United States: introduction of a sequential vaccination schedule of inactivated poliovirus vaccine followed by oral poliovirus vaccine. Recommendations of the Advisory Committee on Immunization Practices (ACIP). $M M W R$ Recomm. Rep. 46(RR-3), 1-25 (1997).

23 Epidemiology and prevention of vaccinepreventable diseases (The Pink Book). In: Epidemiology and Prevention of VaccinePreventable Diseases. Atkinson W, Wolfe C, Hamborsky J (Eds). Centers for Disease Control and Prevention, GA, USA, 249-261 (2012).

24 Vidor E, Meschievitz C, Plotkin S. Fifteen years of experience with Vero-produced enhanced potency inactivated poliovirus vaccine. Pediatr. Infect. J. 16(3), 312-322 (1997).

25 World Health Organization. Global Advisory Committee on Vaccine Safety, 11-12 December 2013. Wkly Epidemiol. Rec. 7(89), 53-60 (2014).

26 Maglione MA, Das L, Raaen L et al. Safety of vaccines used for routine immunization of US children: a systematic review. Pediatrics 134(2), 325-337 (2014).

27 Robertson SE, Traverso HP, Drucker JA et al. Clinical efficacy of a new, enhanced-potency, inactivated poliovirus vaccine. Lancet 1(8591), 897-899 (1988).

28 Moriniere BJ, Van Loon FPL, Rhodes PH et al. Immunogenicity of a supplemental dose of oral versus inactivated poliovirus vaccine. Lancet 341(8860), 1545-1550 (1993).

29 Estivariz CF, Jafari H, Sutter RW et al. Immunogenicity of supplemental doses of poliovirus vaccine for children aged 6-9 months in Moradabad, India: a community-based, randomised controlled trial. Lancet Infect. Dis. 12(2), 128-35 (2012).

30 John J, Giri S, Karthikeyan AS et al. Effect of a single inactivated poliovirus vaccine dose on intestinal immunity against poliovirus in children previously given oral vaccine: an open-label, randomised controlled trial. Lancet 384(9953), 1505-1512 (2014).

31 Jafari H, Deshpande JM, Sutter RW et al. Efficacy of inactivated poliovirus vaccine in India. Science 345(6199), 922-925 (2014). 
32 Meeting of the Strategic Advisory Group of Experts on immunization, November 2013 - conclusions and recommendations. Wkly Epidemiol. Rec. 89(1), 1-20 (2014).

33 Polio vaccines: WHO position paper, January 2014. Wkly Epidemiol. Rec. 89(9), 73-92 (2014).

34 Estívariz CF, Molnár Z, Venczel L et al. Paralytic poliomyelitis associated with Sabin monovalent and bivalent oral polio vaccines in Hungary. Am. J. Epidemiol. 174(3), 316-325 (2011).

35 Kapusinszky B, Molnar Z, Szomor KN, Berencsi G. Molecular characterization of poliovirus isolates from children who contracted vaccine-associated paralytic poliomyelitis (VAPP) following administration of monovalent type 3 oral poliovirus vaccine in the 1960s in Hungary. FEMS Immunol. Med. Microbiol. 58(2), 211-217 (2010).

36 Cáceres VM, Sutter RW. Sabin monovalent oral Polio vaccines: review of past experiences and their potential use after polio eradication. Clin. Infect. Dis. 33(4), 531-541 (2001).

37 Dömök I. Experiences associated with the use of live poliovirus vaccine in Hungary, 1959-1982. Rev. Infect. Dis. 6(Suppl. 2), S413-S418 (1984).

38 Alexander L, Seward JF, Santibanez TA et al. VAccine policy changes and epidemiology of poliomyelitis in the united states. JAMA 292(14), 1696-1701 (2004).

39 Modlin JF, Halsey NA, Thoms ML, Meschievitz CK, Patriarca PA. Humoral and mucosal immunity in infants induced by three sequential inactivated poliovirus vaccine-live attenuated oral poliovirus vaccine immunization schedules. Baltimore Area Polio Vaccine Study Group. J. Infect. Dis. 175(Suppl. 1), S228-S234 (1997).

40 Asturias EJ, Dueger EL, Omer SB et al. Randomized trial of inactivated and live polio vaccine schedules in Guatemalan infants. J. Infect. Dis. 196(5), 692-698 (2007).

41 Faden H, Modlin JF, Thoms ML, McBean AM, Ferdon MB, Ogra PL. Comparative evaluation of immunization with live attenuated and enhanced-potency inactivated trivalent poliovirus vaccines in childhood: systemic and local immune responses. J. Infect. Dis. 162(6), 1291-1297 (1990).

42 Okayasu H, Sutter RW, Czerkinsky C, Ogra PL. Mucosal immunity and poliovirus vaccines: impact on wild poliovirus infection and transmission. Vaccine 29(46), 8205-8214 (2011).
43 Grassly NC, Jafari H, Bahl S et al. Mucosal immunity after vaccination with monovalent and trivalent oral poliovirus vaccine in India. J. Infect. Dis. 200(5), 794-801 (2009).

44 Du Châtelet IP, Merchant AT, Fisher-Hoch S et al. Serological response and poliovirus excretion following different combined oral and inactivated poliovirus vaccines immunization schedules. Vaccine 21(15), 1710-1718 (2003).

45 Anand A, Pallansch MA, Estivariz CF, Gary $\mathrm{H}$, Wassilak SGF. Estimating the likely coverage of inactivated poliovirus vaccine in routine immunization: evidence from demographic and health surveys. J. Infect. Dis. 210 (Suppl. 1), S465-S474 (2014).

46 Martin J. Consequences of the introduction of the new inactivated poliovirus vaccine into the Expanded Programme on Immunization. Rev. Infect. Dis. 6(Suppl. 2), S480-S482 (1984).

47 Duintjer Tebbens RJ, Pallansch MA, Chumakov KM et al. Review and assessment of poliovirus immunity and transmission: synthesis of knowledge gaps and identification of research needs. Risk Anal. 33(4), 606-646 (2013).

48 Duintjer Tebbens RJ, Pallansch MA, Chumakov KM et al. Expert review on poliovirus immunity and transmission. Risk Anal. 33(4), 544-605 (2013).

49 Mangal TD, Aylward RB, Grassly NC. The potential impact of routine immunization with inactivated poliovirus vaccine on wild-type or vaccine-derived poliovirus outbreaks in a posteradication setting. Am. J. Epidemiol. 178(10), 1579-1587 (2013).

50 Wahjuhono G, Revolusiana, , Widhiastuti D et al. Switch from oral to inactivated poliovirus vaccine in Yogyakarta Province, Indonesia: summary of coverage, immunity, and environmental surveillance. J. Infect. Dis. 210(Suppl. 1), S347-S352 (2014).

51 Immunogenicity of 1 or 2 doses of bOPV in Chilean infants primed with IPV vaccine. www.clinicaltrials.gov/ct2/show/NCT018416

52 Safety and immunogenicity of 1 or 2 doses of IPV in Latin American infants primed with bivalent OPV vaccine. www.clinicaltrials.gov/ct2/show/NCT018310

53 John J. Comparative evaluation of immunogenicity and reactogenicity of bivalent oral poliovirus vaccine (bOPV) and trivalent oral poliovirus vaccine ( $\mathrm{tOPV}$ ) in the standard EPI schedule, with or without inactivated polio vaccine (IPV) administration at DTP3 contact: A randomized controlled trial. CTRI/2013/06/003722. http://ctri.nic.in/Clinicaltrials
54 Immunogenicity of inactivated and live Polio vaccines. www.clinicaltrials.gov/ct2/show/NCT018136

55 John J. Duration of Intestinal immunity following inactivated poliovirus vaccine in OPV primed children. CTRI/2014/09/004979. http://ctri.nic.in/Clinicaltrials/showallp.php

56 IPV Clinical Trial - The Gambia. www.clinicaltrials.gov/ct2/show/NCT01847

57 Salk J, Cohen H, Fillastre C et al. Killed poliovirus antigen titration in humans. Dev. Biol. Stand. 41, 119-132 (1977).

58 Salk J, Van Wezel AL, Stoeckel P et al. Theoretical and practical considerations in the application of killed poliovirus vaccine for the control of paralytic poliomyelitis. Dev. Biol. Stand. 47, 181-198 (1980).

59 Salk J, Stoeckel P, Van Wezel AL, Lapinleimu K, Van Steenis G. Antigen content of inactivated poliovirus vaccine for use in a one-or two-dose regimen. Ann. Clin. Res. 14(5-6), 204-212 (1982).

60 World Health Organization. Report from WHO Expert Committee on Biological Standardization. http://whqlibdoc.who.int/trs/WHO

61 Safety study of a single dose of monovalent high-dose inactivated Poliovirus type 2 vaccine ( $m$-IPV2 HD) in infants early in life. www.clinicaltrials.gov/ct2/show/NCT021111

62 Geert Leroux- Roels. Phase 1 Study on the safety and reactogenicity of a single dose of monovalent high-dose inactivated Poliovirus type 2 Vaccine (m-IPV2 HD) given intramuscularly compared to standard trivalent inactivated Poliovirus vaccine (IPV) in healthy adults. http://clinicaltrials.gov/show/NCT01997632

63 Verdijk P, Rots NY, van Oijen MGCT et al. Safety and immunogenicity of a primary series of Sabin-IPV with and without aluminum hydroxide in infants. Vaccine 32(39), 4938-4944 (2014).

64 Prausnitz MR, Mikszta JA, Cormier M, Andrianov AK. Microneedle-based vaccines. Curr. Top. Microbiol. Immunol. 333, 369-393 (2009).

65 Engert J. Implants as sustained release delivery devices for vaccine antigens. In: Subunit Vaccine Delivery. Foged C, Rades T, Perrie Y, Hook S (Eds). Springer NY, USA, 221-241 (2015).

66 Schoellhammer CM, Blankschtein D, Langer R. Skin permeabilization for transdermal drug delivery: recent advances and future prospects. Expert Opin. Drug Deliv. 11(3), 393-407 (2014). 
67 Macadam AJ, Ferguson G, Stone DM et al. Rational design of genetically stable, liveattenuated poliovirus vaccines of all three serotypes: relevance to poliomyelitis eradication. J. Virol. 80(17), 8653-8663 (2006).

68 Toyoda H, Yin J, Mueller S, Wimmer E, Cello J. Oncolytic treatment and cure of neuroblastoma by a novel attenuated poliovirus in a novel poliovirus-susceptible animal model. Cancer Res. 67(6), 2857-2864 (2007).

69 Lauring AS, Jones JO, Andino R. Rationalizing the development of live attenuated virus vaccines. Nat. Biotechnol. 28(6), 573-579 (2010).

70 Burns CC, Campagnoli R, Shaw J, Vincent A, Jorba J, Kew O. Genetic inactivation of poliovirus infectivity by increasing the frequencies of $\mathrm{CpG}$ and $\mathrm{UpA}$ dinucleotides within and across synonymous capsid region codons. J. Virol. 83(19), 9957-9969 (2009).

71 Burns CC, Shaw J, Campagnoli R et al. Modulation of poliovirus replicative fitness in HeLa cells by deoptimization of synonymous codon usage in the capsid region. J. Virol. 80 (7), 3259-3272 (2006).

72 Coleman JR, Papamichail D, Skiena S, Futcher B, Wimmer E, Mueller S. Virus attenuation by genome-scale changes in codon pair bias. Science 320(5884), 1784-1787 (2008).

73 De Jesus N, Franco D, Paul A, Wimmer E, Cello J. Mutation of a single conserved nucleotide between the cloverleaf and internal ribosome entry site attenuates poliovirus neurovirulence. J. Virol. 79(22), 14235-14243 (2005)

74 Vignuzzi M, Wendt E, Andino R. Engineering attenuated virus vaccines by controlling replication fidelity. Nat. Med. 14(2), 154-161 (2008).

75 The polio pipeline - a quarterly update of ongoing research in the Global Polio Eradication Initiative. www.polioeradication.org/Portals

76 Heymann DL, Sutter RW, Aylward RB. A global call for new polio vaccines. Nature 434(7034), 699-700 (2005).
77 Hawken J, Troy SB. Adjuvants and inactivated polio vaccine: a systematic review. Vaccine 30 (49), 6971-6979 (2012).

78 Garcon N, Hem S, Friede M. Evolution of adjuvants across the centuries. In: Vaccines (6th Edition). Plotkin SA, Offit PA, Orenstein W (Eds). W.B. Saunders, London, UK, 58-70 (2013).

79 Ivanov AP, Dragunsky EM, Chumakov KM. 1,25-dihydroxyvitamin $\mathrm{d} 3$ enhances systemic and mucosal immune responses to inactivated poliovirus vaccine in mice. J. Infect. Dis. 193(4), 598-600 (2006).

80 Lycke N. Recent progress in mucosal vaccine development: potential and limitations. Nat. Rev. Immunol. 12(8), 592-605 (2012).

81 Summerton NA, Welch RW, Bondoc L et al. Toward the development of a stable, freeze-dried formulation of Helicobacter pylori killed whole cell vaccine adjuvanted with a novel mutant of Escherichia coli heat-labile toxin. Vaccine 28(5), 1404-1411 (2010).

82 Nelson KS, Janssen JM, Troy SB, Maldonado $Y$. Intradermal fractional dose inactivated polio vaccine: a review of the literature. Vaccine 30 (2), 121-125 (2012).

83 Nirmal S, Cherian T, Samuel BU, Rajasingh J, Raghupathy P, Jacob John T. Immune response of infants to fractional doses of intradermally administered inactivated poliovirus vaccine. Vaccine 16(9), 928-931 (1998).

84 Simoes EF, Padmini B, Steinhoff MC, Jadhav $\mathrm{M}$, John T. ANtibody response of infants to two doses of inactivated poliovirus vaccine of enhanced potency. Am. J. Dis. Child. 139(10), 977-980 (1985).

85 Cadorna-Carlos J, Vidor E, Bonnet MC. Randomized controlled study of fractional doses of inactivated poliovirus vaccine administered intradermally with a needle in the Philippines. Int. J. Infect. Dis. 16(2), e110-e116 (2012).

86 Mohammed AJ, AlAwaidy S, Bawikar S et al. Fractional doses of inactivated poliovirus vaccine in Oman. N. Engl. J. Med.362(25), 2351-2359 (2010).

87 Resik S, Tejeda A, Lago PM et al. Randomized controlled clinical trial of fractional doses of inactivated poliovirus vaccine administered intradermally by needle-free device in Cuba. J. Infect. Dis. 201(9), 1344-1352 (2010).

88 Lago PM, Cáceres VM, Galindo MA et al. Persistence of vaccine-derived poliovirus following a mass vaccination campaign in Cuba: implications for stopping polio vaccination after global eradication. Int. J. Epidemiol. 30(5), 1029-1034 (2001).

89 Norman JJ, Arya JM, McClain MA, Frew PM, Meltzer MI, Prausnitz MR. Microneedle patches: usability and acceptability for self-vaccination against influenza. Vaccine 32(16), 1856-1862 (2014).

90 Van Damme P, Oosterhuis-Kafeja F, Van der Wielen M, Almagor Y, Sharon O, Levin Y. Safety and efficacy of a novel microneedle device for dose sparing intradermal influenza vaccination in healthy adults. Vaccine 27(3), 454-459 (2009).

91 van der Maaden K, Trietsch SJ, Kraan H et al. Novel hollow microneedle technology for depth-controlled microinjection-mediated dermal vaccination: a study with polio vaccine in rats. Pharm. Res. 31, 1846-1854 (2014).

92 Correia-Pinto JF, Csaba N, Alonso MJ. Vaccine delivery carriers: insights and future perspectives. Int. J. Pharm. 440(1), 27-38 (2013).

93 Bakker WA, Thomassen YE, van't Oever AG et al. Inactivated polio vaccine development for technology transfer using attenuated Sabin poliovirus strains to shift from Salk-IPV to Sabin-IPV. Vaccine 29(41), 7188-7196 (2011).

94 Weekly Epidemiological Record. Meeting of the Strategic Advisory Group of Experts on Immunization, April 2014 - conclusions and recommendations. World Health Organization. Wkly Epidemiol. Rec. 89(21), 221-236 (2014). 